\title{
Co-axial depth map sensor with an extended depth range
}

\author{
Mohan Xu, Hong Hua
}

Mohan Xu, Hong Hua, "Co-axial depth map sensor with an extended depth range," Proc. SPIE 11040, Optical Design Challenge 2019, 1104003 (27

February 2019); doi: 10.1117/12.2523653

SPIE Event: SPIE Optical Design Challenge, 2019, San Francisco, California, United States 


\title{
Co-axial depth map sensor with an extended depth range
}

\author{
Mohan $\mathrm{Xu}^{*}$, Hong Hua
}

3D Visualization and Imaging Systems Laboratory, College of Optical Sciences, University of Arizona, 1630 East University Boulevard, Tucson 85721, Arizona, USA

\begin{abstract}
The conventional depth map sensors have limited depth range, or they need to sacrifice depth accuracy for a larger working range. To overcome such problems, in this work, we propose a co-axial depth map sensor with an extended depth range based on controlled aberrations. This depth map sensor implements depth measurement by projecting a near-infrared astigmatic pattern onto the test scene and measuring the contrast change of the reflected pattern image in the tangential and sagittal directions. By adding a tunable lens in the projection optics, this depth map sensor can achieve the extended depth measurement without the loss of high depth accuracy and high depth map resolution.
\end{abstract}

Keywords: Depth map sensor, 3D camera, controlled aberration, extended depth range, focus tunable lens

\section{INTRODUCTION}

Virtual reality and augmented reality consumer electronics are powered by the 3D depth sensors that can scan and digitalize a user's the environment in the real time. The captured depth map provides numerous potential applications for VR/AR system such as the 3D modeling for the display content or the interaction with the display through the user gestures.

Currently, the most popular technologies used in the depth map sensors are the passive stereo ${ }^{1}$, time of flight ${ }^{2-7}(\mathrm{ToF})$ and structured light ${ }^{8}$. Both the passive stereo and structured light depth sensing are based on the triangulation method which requires the baseline separation between the dual imaging paths or between the projection path and imaging path. Among those technologies, the ToF method has the largest depth measurement range but lower resolution. To enlarge the depth range while keeping the high resolution, in this paper, we propose a co-axial depth map sensor with an extended depth range.

The main contributions of the depth sensor described in this paper include

1. It can extend depth range for measurement while maintaining the high depth map resolution

2. It has a co-axial design for its projection path and imaging path, which can potentially yield a system being more compact and cost-effective than conventional structured light depth cameras.

3. Its imaging path can provide a high-resolution 2D image along with a 3D depth map. The 2D images can be used to improve the accuracy of the 3D map.

In this paper, we introduce the concept of this extended depth range co-axial depth sensor in section 2 . The optical design results are presented in section 3 . We then demonstrate the depth measurement simulation for different target distance in section 4 .

\section{CONCEPT}

This depth map sensor works based on the controlled aberration method ${ }^{9}$. It implements the depth measurement by projecting an astigmatic pattern on the test scene and then measuring the contrast change of the reflected pattern image in the tangential and sagittal directions. There is no separation required between the projection path and the imaging path. Therefore, this depth map sensor could be co-axial for more compact size and lower budget. Furthermore, by adding a tunable lens in the projection optics, the depth map sensor can achieve the extended depth measurement without the loss of high depth accuracy and high depth map resolution.

As shown in Fig.1, in this design, the projection path and imaging path are combined by a beam splitter to form a co-axial design. For the projection path, light emitted from a near-infrared LED is collimated and then encoded by a DMD chip 
(DLP4500NIR ${ }^{10}$ ) with the designed projection pattern. The encoded light then passes through a cylindrical lens which adds extra power to it in one direction, namely the extraordinary projection direction, and its orthogonal direction is the ordinary projection direction. The output light is then projected through the projection optics onto a target scene. Then the reverse projection optics works as an imaging lens to form a pattern image for the reflected light through the beam splitter onto an image sensor. Due to astigmatism, the projected light forms a tangential focus and a sagittal focus at two different axial depths. For a test plane moving in the range $\Delta \mathrm{z}$ from the tangential focus to the sagittal focus, the image contrast of reflected pattern in the extraordinary projection direction keeps decreasing along the optical axis while the contrast in the ordinary projection direction keeps increasing. Therefore, by measuring the reflected image contrast in those two orthogonal directions, we can get the depth information for the test object.

For a depth map sensor with fixed projection lens power, the distance $\Delta \mathrm{z}$ defines its depth measurement range and it is determined by the cylindrical lens power added. In order to extend the depth range, we could insert an electronically focus tunable lens into the projection optics. Then the problem is to identify the location and power of the focus tunable lens. As described above, in the co-axial configuration, the reverse projection optics works as the imaging optics. With the power change of the tunable lens, we want to shift the location of the DMD optical conjugate plane accordingly but maintain the image size on the sensor. In this sense, the imaging optics must be telecentric in the image space and the focus tunable lens is placed at the system stop which could keep the telecentricity. With this structure, when tuning the tunable lens, the depth measurement range $\Delta \mathrm{z}$ scanning along the optical axis which enables an extended depth measurement range of the proposed depth map sensor.

Moreover, if we use an RGB-NIR image sensor in the system, such as the Imec RGB-NIR image sensor ${ }^{11}$. The RGB channel can be used to capture the 2D image of the test scene while the near-infrared image is used to create the depth map.

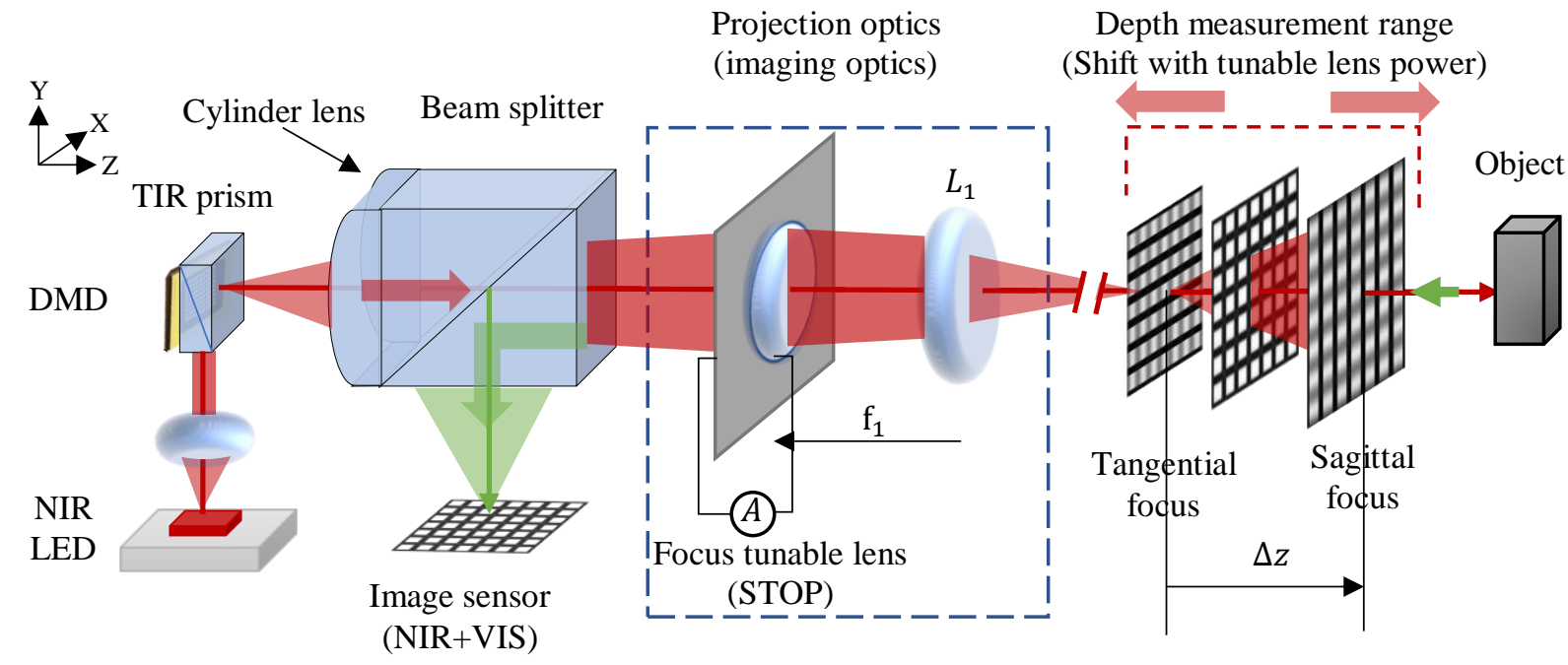

Figure1. The concept of the co-axial depth map sensor with an extended depth range

\section{OPTICAL DESIGN}

In the optical design of this co-axial depth map sensor, it can be separated into the imaging path design and the projection path design. In the imaging path design, the difficult parts are the telecentricity in the image space and the optimization of the imaging lens for the different power of the focus tunable lens. Then for the projection path, based on the co-axial design, the projection lens is the reverse of the imaging lens thus the projection path design mainly focusses on investigating the power of the cylindrical lens and its corresponding depth range $\Delta \mathrm{z}$.

\subsection{The imaging path design}

For the imaging lens design, the first step is to design an imaging lens with the exit pupil at infinity, then insert a focus tunable lens where its adaptive surface coincides with the system stop. In this design, we use the tunable lens optotune 
EL-16-40-TC-VIS-5D ${ }^{12}$ to enlarge the depth range. Figure 2 (a) shows the design layout for the imaging path. The reflected light after the beam splitter is unfolded in the figure for convenience.

(a)

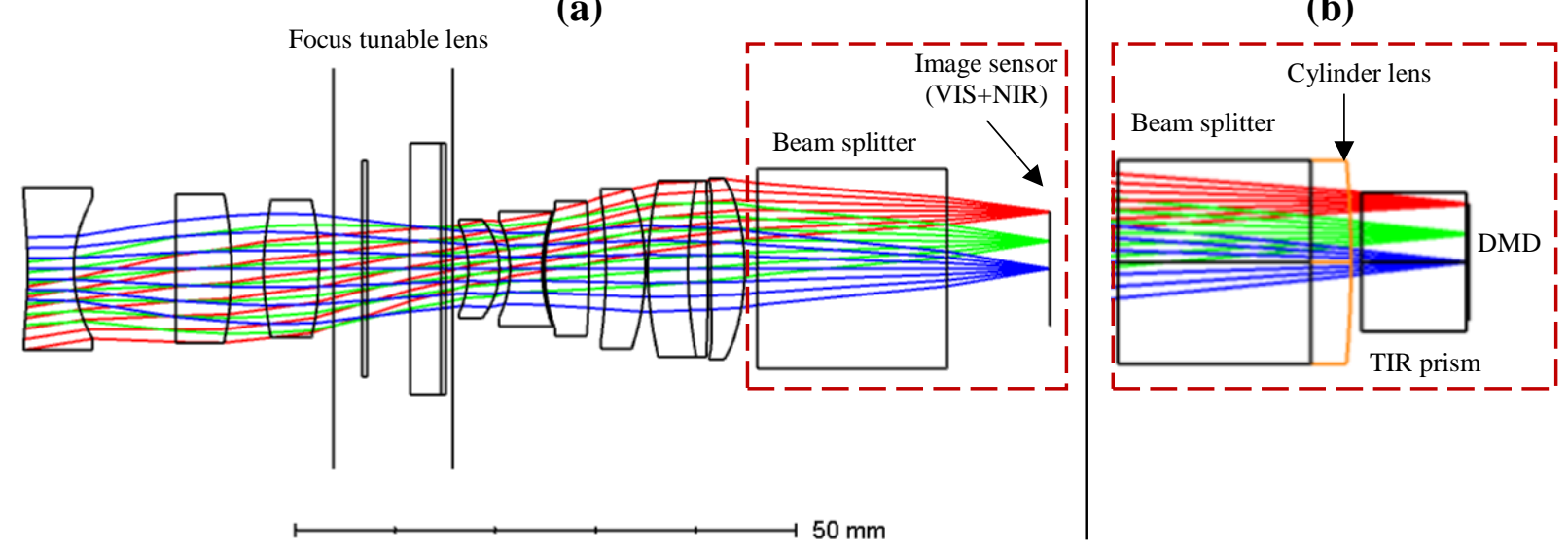

Figure 2. (a) is the imaging path layout (b) is the layer out of the projection path for the part

different with the imaging path

Table 1 summarizes the imaging path specification. The FOV of the system is $31.2^{\circ}$, while the image space F/\# is 3 . We use the $0.486 \mathrm{um}, 0.588 \mathrm{um}, 0.656 \mathrm{um}$ for the VIS wavelength and the $0.81 \mathrm{um}$ for the near infrared working wavelength of the NIR DMD chip. For the focus tunable lens, the powers are designed as 0.5 diopter, 0 diopters and -0.35 diopter. With the tunable, it shifts the object distance of the imaging path into $550 \mathrm{~mm}, 1000 \mathrm{~mm}, 2000 \mathrm{~mm}$ to represent the near and middle and far working range respectively. For all the designed tunable lens power, the exit pupil positions are all far from the system so that we can treat the imaging lens image space telecentric.

Table 1. Imaging path specification

\begin{tabular}{|c|c|c|c|}
\hline & FOV & $\begin{array}{l}\text { Image space } \\
\text { F/\# }\end{array}$ & Wavelengths \\
\hline & $\pm 15.6^{\circ}$ & 3 & $\begin{array}{l}\text { F,d,c (VIS), } \\
\text { 0.810um (NIR) }\end{array}$ \\
\hline \multicolumn{4}{|c|}{ Parameters change with tunable lens (TL) } \\
\hline Depth Range & Near & Middle & Far \\
\hline $\operatorname{TL} \operatorname{CVR}(1 / \mathrm{mm})$ & $1.709 \times 10^{-3}$ & 0 & -0.0012 \\
\hline TL power (diopter) & 0.5 & 0 & -0.35 \\
\hline Object distance (mm) & 550 & 1000 & 2000 \\
\hline $\mathrm{XP}$ position $(\mathrm{mm})$ & $1.4 \mathrm{e}+5$ & $1 e+10$ & $-1.7 e+5$ \\
\hline Paraxial Magnification & -0.034 & -0.019 & -0.0096 \\
\hline
\end{tabular}

For the tunable lens power change, the imaging path shifts the conjugate plane of the image sensor accordingly. For those three chosen working distances, optimize the imaging lens design and then plot the MTF for imaging path performance in Figure (3). At the frequency 35 cycles/mm, the MTF value is all higher than 0.4 . 

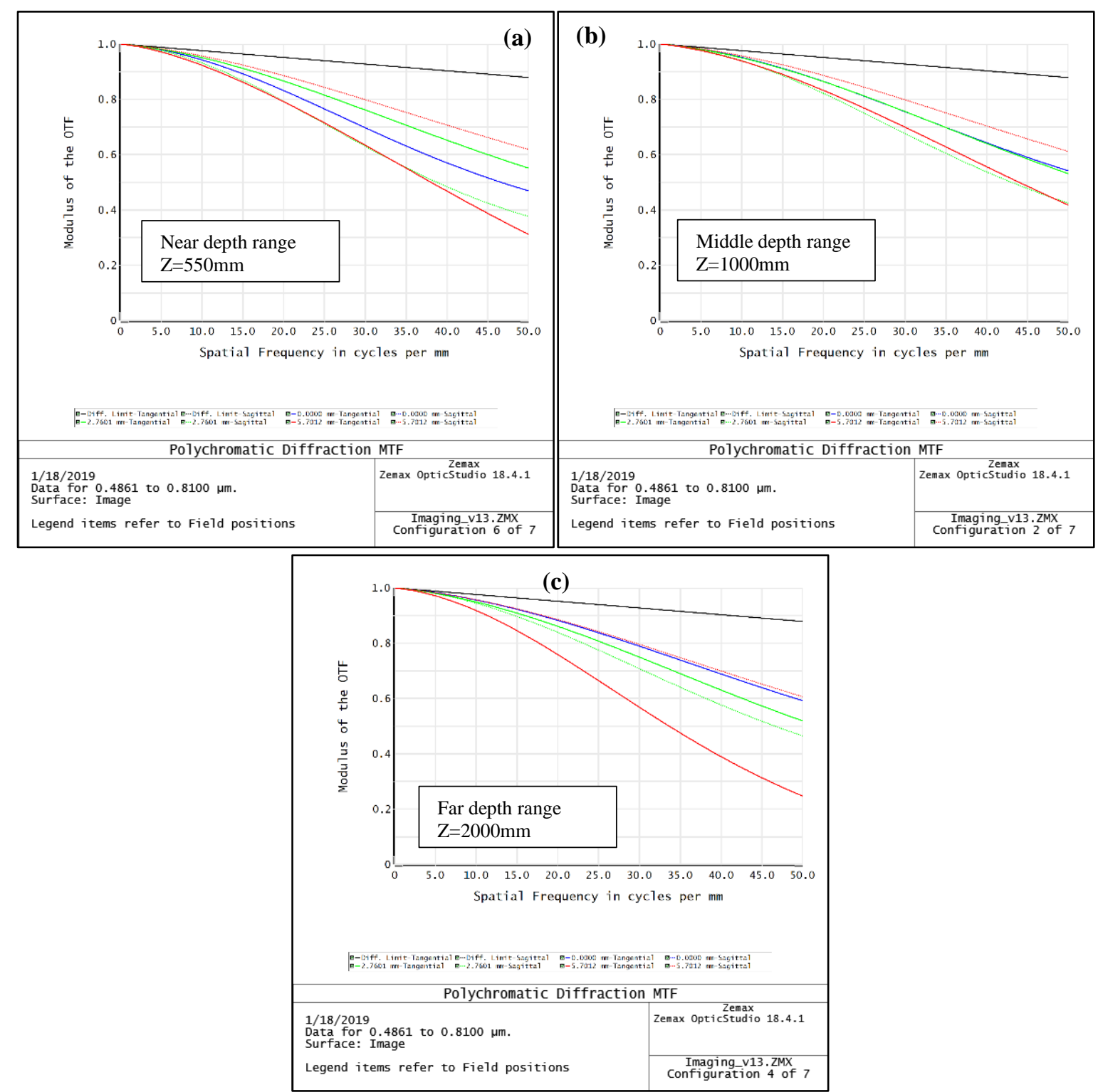

Figure 3. The polychromatic MTF for (a) near depth range, while the object position is $550 \mathrm{~mm}$ and tunable lens power, is 0.5 diopter, (b) middle depth range while the object distance is $1000 \mathrm{~mm}$ and tunable lens power is 0 diopter, (c) the far depth range while the object distance is $2000 \mathrm{~mm}$ and the tunable lens power is -0.35 diopter. 


\subsection{The projection path design}

As described above, the projection optics is the reverse of the imaging optics. Therefore, we only show the layout of the difference between the projection path and the image path in the red dash box in the Figure2. (b). In the projection path, the projection distance is much larger than the object distance; normally we switch the object and image in the optical design. Therefore we still treat it as a camera lens for design and the DMD is the image plane. As shown in the Figure2. (b), there is a cylindrical lens to introduce extra power for the extraordinary projection direction and a TIR prism in front of the DMD chip to coupling the illumination. The TIR prism is treated as a Plane-Parallel Plate here. In this sense, the critical point of this subsection is to design the cylinder lens power and the corresponding depth measurement range under the different focus tunable lens power. For the middle range, the ordinary projection direction has the conjugate plane of the DMD is at $1000 \mathrm{~mm}$, for the extraordinary projection direction by adding 5.885 diopters cylinder the projection path forms a tangential focus at $500 \mathrm{~mm}$. Table 2 shows the tangential and sagittal focus location for different tunable lens power.

Table 2. The parameter of the projection path

\begin{tabular}{|l|l|l|c|}
\hline Cylinder lens power (diopter) & \multicolumn{3}{|c|}{5.8846} \\
\hline Depth range & Near & Middle & Far \\
\hline Tunable lens power (diopter) & 0.5 & 0 & -0.35 \\
\hline Tangential focus distance (mm) & 340 & 500 & 700 \\
\hline Sagittal focus distance (mm) & 550 & 1000 & 2000 \\
\hline
\end{tabular}

\section{DEPTH MEASUREMENT SIMULATION}

In this section, we demonstrate the depth measurement ability with the designed depth sensor by shifting a test screen along the optical axis at different distance and projecting a pattern onto the screen and then capturing the image of the reflected projection pattern. As shown in Figure 4, the overall extended depth range is constructed with three sub-ranges. The specific distances are calculated above and summarized in Table 2. For each subrange, we then illustrate that when moving the test screen away from the depth sensor, the image contrast of the reflected pattern in the extraordinary projection direction decreases while the contrast in ordinary projection direction increases. Then by checking the contrast change for all the depth sub-ranges we could prove that the measurement works in an extended depth range.

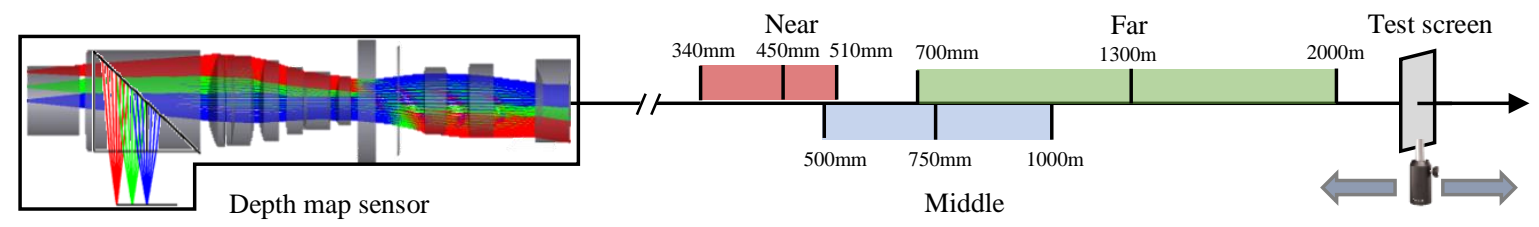

Figure 4. The setup for the depth measurement simulation

In this simulation, we use the USAF resolution target as the projection pattern to prove the depth measurement concept. The reason is that the USAF resolution target is constructed with orthogonal line pairs of different spatial frequencies. It is straightforward to see the contrast change of the reflected pattern image.

The reflected pattern images are illustrated in Figure 5. Each row of the figure represents a sub depth range. The left column shows the image for the tangential focal plane location and the right column is the sagittal focal plane location. The image contrast of the tangential direction decreases monotonically from the left column to the right column, meanwhile, the image contrast of the sagittal direction increases. Specially, for the line pairs in the red dash box and yellow dash box, the contrast changes significantly along the optical axis at the sampled depth.

Due the contrast change described above, there is a unique value of the contrast ratio between the tangential and sagittal direction corresponds to each depth. The contrast change with depth follows the same trend in all the sub-ranges. Therefore, the proposed depth sensor could work in an extended depth range. 

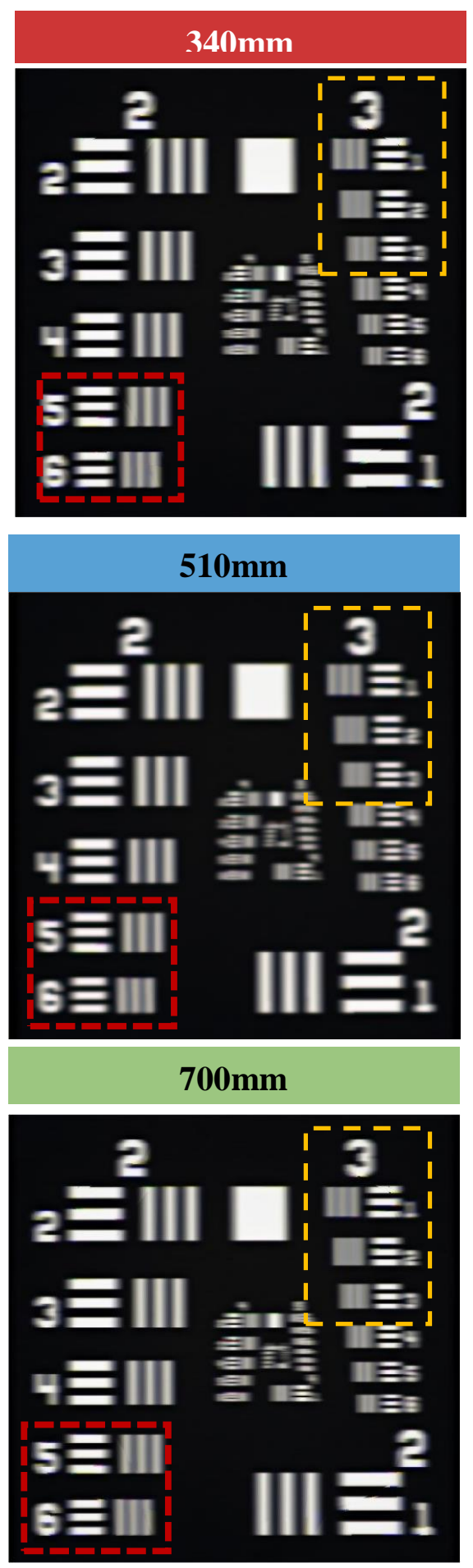
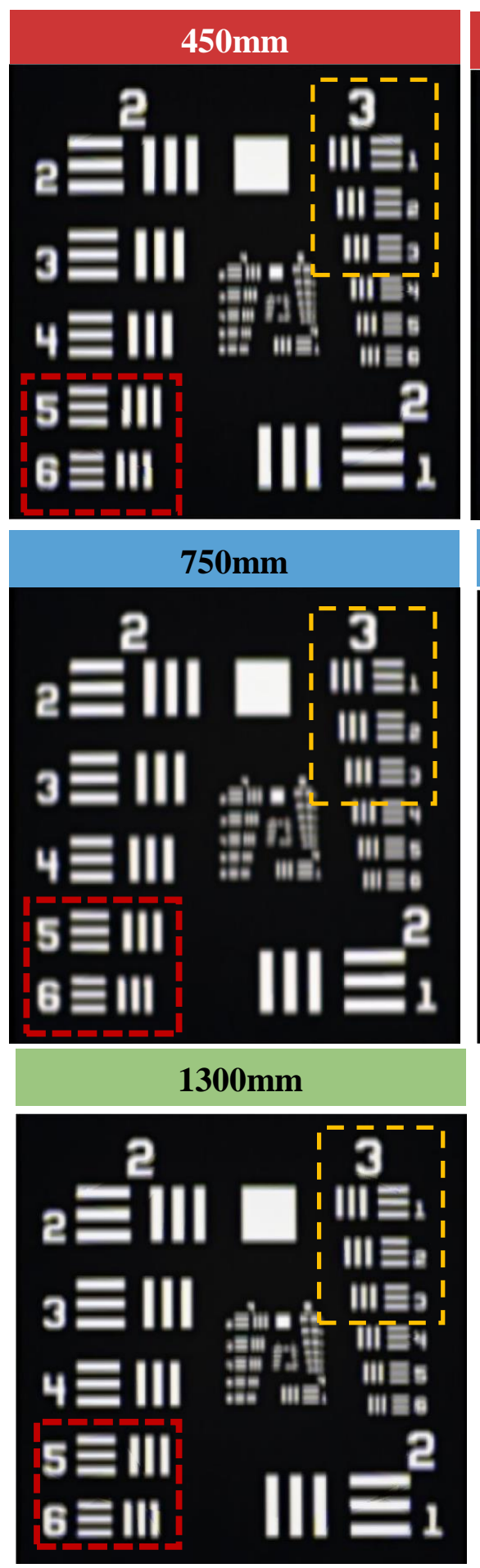
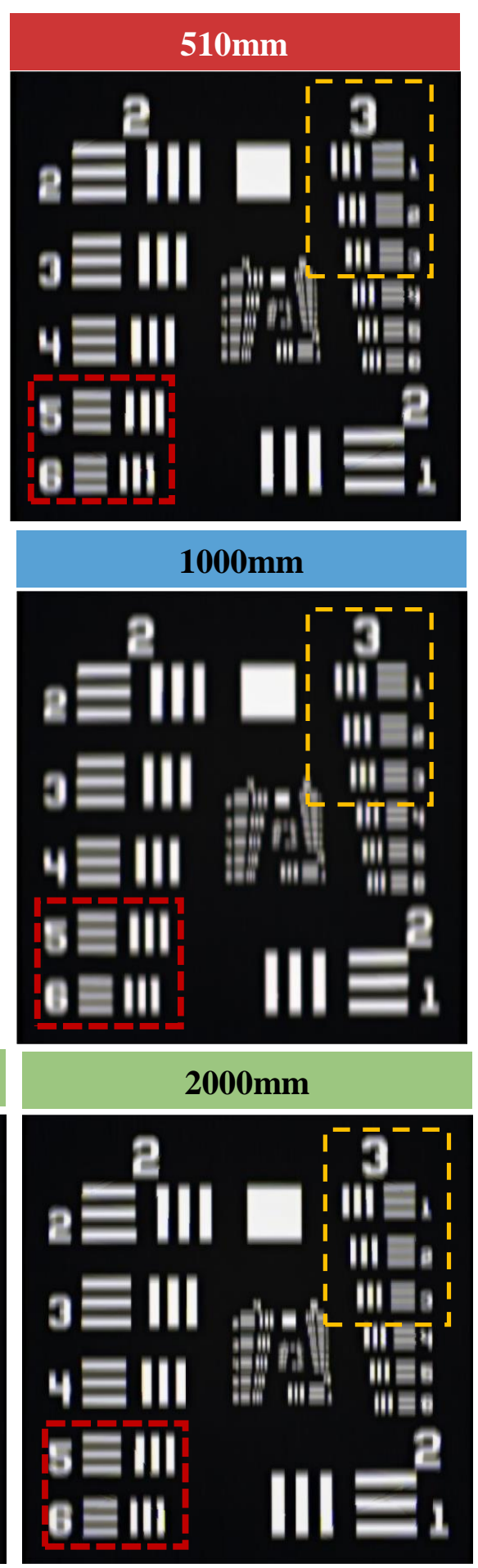

Figure 5. The reflected pattern image for the test plane locates at different distances 
For the group 5 line-pairs in the red dash boxes in Figure 5, calculate the contrast ratio from the reflected pattern image and then plot the result in the dioptric space in Figure 6. The depth range in dioptric space for each sub depth range is the same which is determined by the cylinder lens power, and the overlap between the adjacent sub depth ranges is determined by the power of the focus tunable lens. In this sense, for a target depth measurement range, by carefully design the projection lens and cylinder lens and use the tunable lens, the proposed depth map senor can be adapted for the requirement based on the same principle.

Meanwhile, from Figure 6 also inspire us with a method to improve the depth accuracy. For the overlap area between the sub ranges, we can measurement a depth information in the multiple sub-ranges. Those multiple results could potentially increase the depth measurement accuracy.

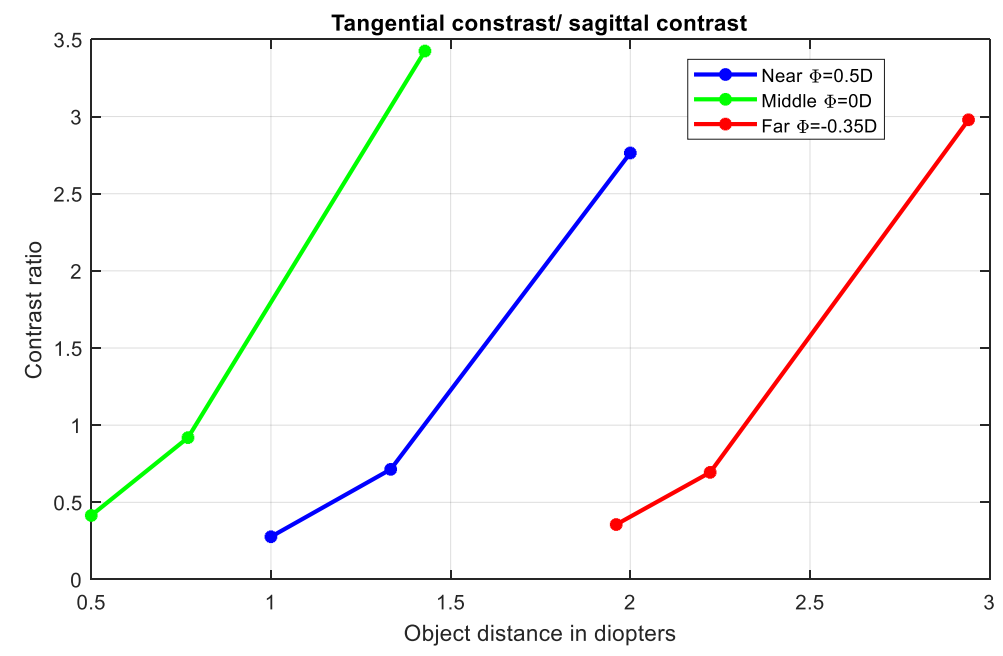

Figure6. The contrast ratio between the tangential direction to the sagittal direction for the reflected projection pattern of the USAF resolution target group 2 part 5 in the dioptric space.

\section{CONCLUSION}

In this paper, we proposed a depth map sensor with an extended depth range. This depth sensor implements depth measurement by projecting an astigmatic pattern on the test scene and then measuring the contrast change of the reflected pattern image in the tangential and sagittal directions. A focus tunable lens is added to the stop of the object space telecentric projection optics to extend the working range. We then present the optical design for the imaging path and the projection path of the system and then prove that the proposed design could be achieve the depth measurement for an extended range by simulating the reflected pattern image and illustrating its contrast change along with the depth chang

\section{REFERENCE}

[1] Davis, J., Ramamoorthi, R. and Rusinkiewicz, S., "Spacetime stereo: A unifying framework for depth from triangulation," Computer Vision and Pattern Recognition, 2003. Proceedings. 2003 IEEE Computer Society Conference, 359 (2003).

[2] Kollorz, E., Penne, J., Hornegger, J. and Barke, A., "Gesture recognition with a time-of-flight camera," International Journal of Intelligent Systems Technologies and Applications 5(3), 334 (2008).

[3] Yahav, G., Iddan, G. J. and Mandelboum, D., "3D Imaging Camera for Gaming Application," ICCE, 1-2 ( 2007).

[4] Lange, R. and Seitz, P., "Solid-state time-of-flight range camera," JQE 37(3), 390-397 (2001).

[5] Kawahito, S., Halin, I. A., Ushinaga, T., Sawada, T., Homma, M. and Maeda, Y., "A CMOS Time-of-Flight Range Image Sensor With Gates-on-Field-Oxide Structure," JSEN 7(12), 1578-1586 (2007).

[6] Ganapathi, V., Plagemann, C., Koller, D. and Thrun, S., "Real time motion capture using a single time-of-flight camera," CVPR, 755-762 (Jun 2010). 
[7] Gokturk, S. B., Yalcin, H. and Bamji, C., "A Time-Of-Flight Depth Sensor - System Description, Issues and Solutions," CVPRW, 35 (2004).

[8] YOEL, A., BARAK, F., MEIR, M. and ALEXANDER, S., "Depth mapping using projected patterns" (US8150142B2) (2012).

[9] Birch, G. C., Tyo, J. S. and Schwiegerling, J., "Depth measurements through controlled aberrations of projected patterns," Optics express 20(6), 6561 (2012).

[10] “DLP4500NIR.”, <http://www.ti.com/product/DLP4500NIR>.

[11] "Imec RGB-NIR image sensor.", <https://www.imec-int.com/en/hyperspectral-imaging>.

[12] “EL-16-40-TC.”, <https://www.optotune.com/products/focus-tunable-lenses/electrical-lens-el-16-40-tc>. 